\title{
Magnetic Polymer Colloids for Ultrasensitive Molecular Imaging
}

Sundas Riaz ${ }^{1}$

Nasir M. Ahmad ${ }^{*}$

Sumera Khizar ${ }^{1}$

Gul Shahnaz ${ }^{2}$

Noureddine Lebaz ${ }^{3}$

Abdelhamid Elaissari ${ }^{4}$

${ }_{1}$ Polymer Research Lab, School of Chemical and Materials Engineering (SCME), National University of Sciences and Technology

(NUST), H-12 Sector, Islamabad-44000, Pakistan

2 Department of Pharmacy, Faculty of Biological Sciences, Quaid-i-Azam University, Islamabad-45320, Pakistan

3 Univ Lyon, University Claude Bernard Lyon-1, CNRS, LAGEPP UMR-5007, 43 boulevard du 11 novembre 1918, F-69100,

Villeurbanne, France

4 Univ Lyon, University Claude Bernard Lyon-1, CNRS, ISA-UMR 5280, F-69622 Lyon, France

*Corresponding author: nasir.ahmad@scme.nust.edu.pk

\begin{abstract}
Magnetic resonance imaging for in vivo diagnosis has been widely used based on numerous contrast agents ranging from molecular size to nanoparticles. This non-invasive technique has been used for various instantaneous in vivo diagnosis via direct imaging of the body to evaluate the state of different organs including spleen, liver, kidneys, pancreas, blood vessels, all possible tumors of chest and abdomen, inflammatory bowel disease and vessel, malformations of the blood vessels and fetus in the womb, etc. Then the aim of this chapter is not only to report the fundamentals and the basis of magnetic resonance imaging, but also the recent developments and utilization in life sciences. In addition, ultrasensitive molecular imaging through the development of combinatorial thin film gradients containing magnetic nanoparticles is reported and described.
\end{abstract}

\section{Keywords}

Magnetic resonance imaging; Ultrasensitive molecular imaging ; Contrast agents ; Thin film gradients ; Iron oxide nanoparticles 


\section{Table of Contents}

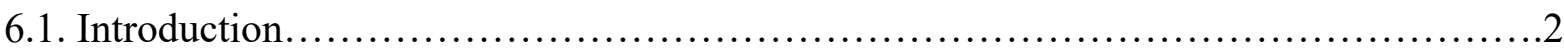

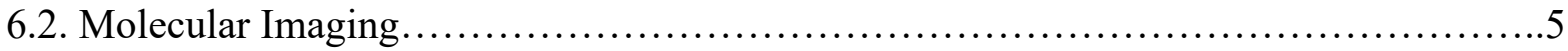

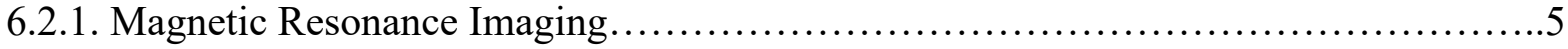

6.2.2. Basic Components of an MRI Machine.............................................

6.2.3. Development of Contrast Agents for MRI..................................... 8

6.3. Development of MRI as a Tool for Ultrasensitive Molecular

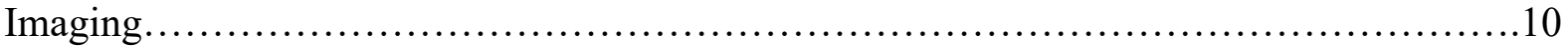

6.3.1. Development of Iron Oxide-Based Contrast Agents for Ultrasensitive Imaging.......11

6.3.2. Development of an Imaging Platform for

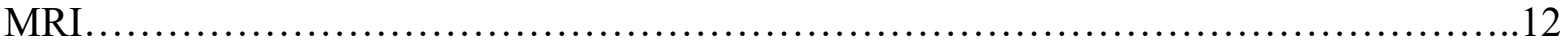

6.3.3. Electrostatic Layer-by-Layer Self Assembly for Magnetic Thin

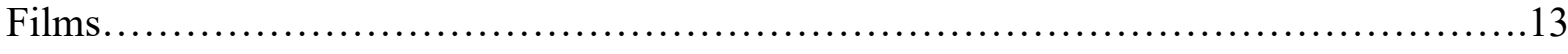

6.4.Conclusions \& Final Remarks

15

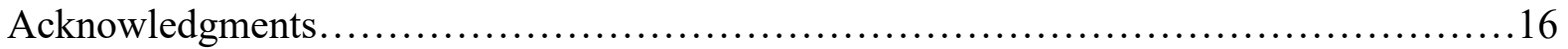

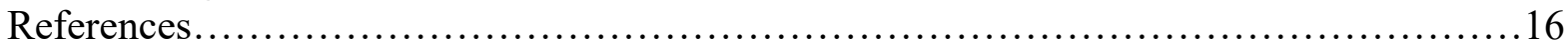

\subsection{Introduction}

Magnetic Resonance Imaging (MRI), based on the concept of nuclear magnetic resonance, has become a popular diagnostic technique since the acquisition of the first clinical MRI scan in 1977. A typical clinical MRI machine uses a primary magnetic field with a magnetic field strength of 1.5 to 3 Tesla (termed as 1.5 or $3 \mathrm{~T}$ ) and specifically designed radiofrequency induction coils to produce highly detailed images (Simonsen et al. 2019). The image acquisition is based on the interaction of hydrogen nuclei associated with water molecules present in the human body, with a radio frequency pulse applied in the presence of a uniform magnetic field. The details in an MR image are a consequence of the contrast generated due to a variation in the amount of water molecules present in different tissues of the body (Mastrogiacomo et al. 2019). MRI is used to detect the nucleus of hydrogen $(\mathrm{H}+)$ in the water molecules as present in the body. Protons are positively charged subatomic particles that are constantly rotating along their axis. The rotational movement of protons in an atom gives rise to a quantum-mechanic atomic property that is called spin. Since this technique uses a magnetic field and electromagnetic radiofrequency rather than ionizing radiation employed in other contemporary molecular imaging techniques like X-ray computed tomography (X-ray CT or CT) and positron emission tomography (PET), it has become a 
gold standard as a considerably safe, non-invasive imaging technique in clinical diagnostics (Atabo and Umar 2019).

Besides its use as an exceptional diagnostic technique, MRI has a diverse assortment of applications in both industry and academia. Some applications of MRI are based on the unique ability of this technique to non-invasively image live subjects and intact objects, whereas various other applications rely on its use as a high-resolution in vitro imaging technique. In the context of imaging of live subjects, MRI is used in the pharmaceutical industry for in vivo theranostic research during pre-clinical trials of new drugs in small animals (Fiordelisi et al. 2019). Most of the currently available diagnosis and therapies are invasive, time-consuming, and associated with severe toxic side effects. Theranostic research refers to the simultaneous administration and observation of the effects of a therapeutic agent or drug in a live animal model in a non-invasive manner (Wong et al. 2020).

The ability of MRI to obtain images solely based on water molecules in a sample has led to the use of MRI as an in vitro characterization technique for studying the hydration effect of materials like cement and concrete to improve the durability and performances of cement-based materials (Du et al. 2019). Similarly, a variety of imaging techniques are currently used within the field of pharmaceutics to help understand and determine a wide range of phenomena associated with drug release from hydrophilic matrix tablets. The hydration effects of tablets are also being studied in the pharmaceutical industry with the help of MRI (Ward et al. 2019).

The use of MRI as an in vitro diagnostic tool for cellular imaging and analysis of ex-vivo histological samples is also a very interesting prospect (Mastrogiacomo et al. 2019). A typical 1.5T clinical MRI machine produces images in the resolution of the millimeter-to-submillimeter range. While this resolution is sufficient to obtain a clinical diagnosis, a relatively limited signal-to-noise ratio becomes a liability in the use of the same system for an in vitro diagnostic application, which requires detail at the level of single cells (Geraldi and Giri-Rachman 2019). Early diagnosis of infectious diseases represents powerful means to increase patient survival rate, avoid disease spreading, and decrease healthcare costs.

Recent scientific progress in the field of magnetic resonance imaging is targeted at improving the sensitivity of MRI in terms of improvement in spatial resolution and signal-to-noise ratio so that the full potential of MRI as an ultrasensitive imaging technique can be reached. These research areas revolve around the following themes:

(i) improvement in instrument design or hardware, like the development of stronger primary magnetic fields up to $11 \mathrm{~T}$, and the development of specialized gradient coils to improve resolution (Zubkov et al. 2018). 
(ii) development of exogenous contrast agents that significantly increase the signal to noise ratio and enhance the sensitivity of MR images by interacting with the magnetic spins of water molecules in a sample. Current trends in the development of ultrasensitive MRI contrast agents focus on the effect of size of contrast agent on its magnetic resonance properties and the significance of polymer-based coatings that may impart unique modalities to the contrast agent like stability or specific functionality. The implementation of the biocompatible surface coating to the inorganic iron core provides a stable behavior under physiological conditions (i.e. inhibits aggregation). A variety of substances include a synthetic and natural polymer (e.g. proteins or dextran) and amphiphilic molecules such as fatty acids or phospholipids which can be used as coating materials. The controlled surface have been increasingly exploited in magnetic resonance imaging (MRI) for contrast enhancement (Khalid et al. 2020).

(iii) another interesting research area involves the development of MRI 'phantoms' that are composed of known quantities of magnetic contrast agents in aqueous solutions or gels that serve as standard test materials for calibration of instruments (Zubkov et al. 2018).

(iv) integration of the concept of nuclear magnetic resonance with other more sensitive techniques like optical microscopy (magnetic resonance microscopy) and atomic force microscopy (magnetic resonance force microscopy) etc. (Grob et al. 2019). This integration allows us to detect MRI signals from nanoscale sample volumes, providing a paradigm-changing potential for structural biology and medical research. Thus far, however, experiments have not reached sufficient spatial resolution for retrieving meaningful structural information from samples.

This chapter focuses on the utilization of MRI as a technique for ultrasensitive molecular imaging through the development of a combinatorial chip that can be used as a substrate for in vitro imaging of fluidic biological samples like blood, or suspensions of cells and cell lysates.

Conventional techniques for cellular imaging through MRI involve a sample preparation regimen where cell samples are mixed with different concentrations of a known contrast agent and placed inside agarose pockets before imaging. A gradient thin film capable of producing a significant contrast difference along the gradient will eliminate the need for such sample preparation steps and allow for a simple and quick surface-based imaging method (Khizar et al. 2020). Thin film gradients using the layer-by-layer technique which can be a promising technique in the future for disposable lab-on-chip as a dipstick approach for ultrasensitive molecular imaging of bioanalytes. The proposed materials chip is composed of a gradient thin film of colloidal core-shell magnetic nanoparticles that serve as exogenous contrast agents to generate a negative contrast in aqueous environments in an MRI (Khizar et al. 2020). The unique core-shell morphology of these colloids coupled with adequately tailored magnetic properties can significantly increase the signal-to-noise 
ratio and consequently, the sensitivity of the MR images. The thin films when used as substrates can serve as a means to effectively image cells without changing the instrument in any way.

The gradient thin films have been fabricated by a very simple self-assembly process that is easily reproducible and allows them to be used as a disposable, lab-on-chip device for a dipstick-like approach towards molecular diagnostics. Elimination of sample preparation steps and a quick, quantifiable response may even point towards the application of such films for point-of-care diagnostics.

\subsection{Molecular Imaging}

Molecular imaging refers to a variety of imaging techniques based on the imaging of specific target molecules. A molecular imaging technique might be capable of directly imaging a target molecule that is indigenously present in a sample or it may rely on the introduction of specifically designed molecular probes or tracers that bind to a target molecule and then indirectly image the molecule bound to the tracer or probe. Some examples of molecular imaging techniques include but are not limited to MRI, X-ray CT, and PET. X-ray CT uses X-rays and PET relies on the use of radioactive tracers to produce high-resolution two- and three-dimensional images. MRI, on the other hand, capitalizes on the nuclear magnetic resonance phenomenon of hydrogen nuclei present in the water molecules in a sample without the use of ionizing radiation or radioactive tracers which makes it a relatively safe, non-invasive imaging technique for clinical diagnostics (Gordon et al.2019). Magnetic resonance imaging is an emerging technology that enables the noninvasive visualization, characterization, and quantification of molecular events within a living subject.

\subsubsection{Magnetic Resonance Imaging}

Magnetic resonance imaging or MRI typically relies on the magnetic properties of hydrogen nuclei that are present in water molecules of the sample as reported before. Although some specialty applications of MRI target other atomic nuclei like phosphorus, sodium, and carbon, hydrogen being the most abundant atom in the human body is usually targeted for imaging. The human body can be considered as an arrangement of cells and tissues with different water contents that make up different types of organs and organ systems. The MR image is therefore a product of differential distribution of these water molecules in a three-dimensional space (Atabo and Umar 2019).

The hydrogen nucleus is a spinning charged species with an associated magnetic field termed as a magnetic moment (Mastrogiacomo et al. 2019). 

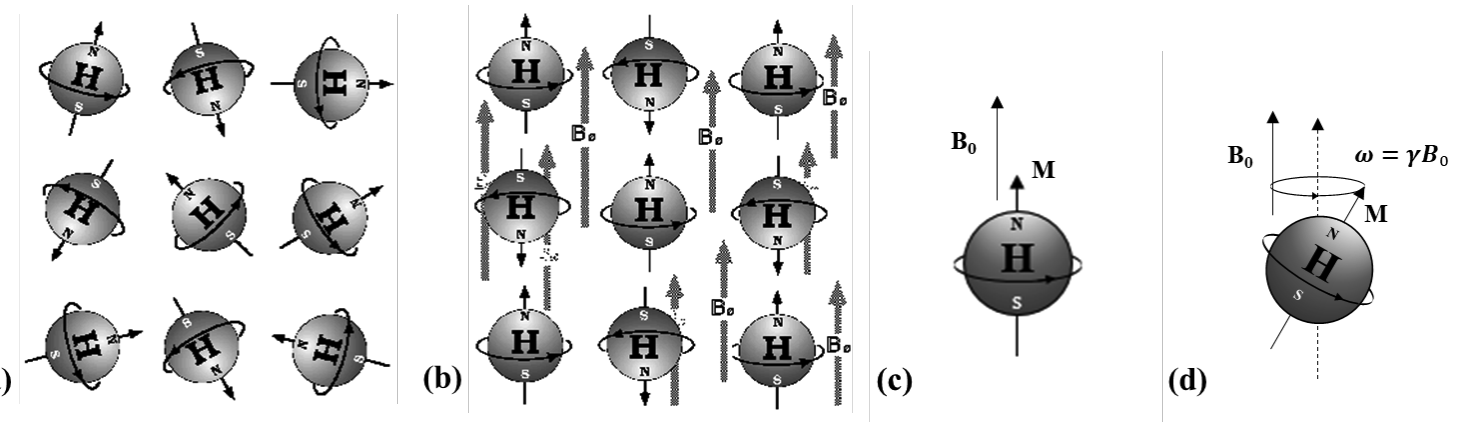

Figure 1

AS shown in Figure 1(a), the spins of hydrogen nuclei in the human body are randomly oriented in the absence of an external magnetic field resulting in a zero net magnetic moment. When an external field is applied, the spins of hydrogen nuclei tend to align in a direction parallel or antiparallel to the applied field to achieve a low energy state (Figure 1(b)). Two main properties of these nuclei should be taken into account to understand their behavior in MR imaging.

(i) at any given time, more nuclei tend to align parallel to the field than against it. As a result, the net magnetic vector is parallel to the applied field. This magnetization is termed as longitudinal magnetization (Mastrogiacomo et al. 2019). (Figure 1(c)).

(ii) the aligned nuclei exhibit a phenomenon called precession (Mastrogiacomo et al. 2019), where the axes of the spins revolve around the axis of the external magnetic field, $\mathrm{B}_{0}$, with a certain frequency, $\omega_{\mathrm{L}}$, termed the Larmor frequency $\left(\omega_{\mathrm{L}}=\gamma \mathbf{B}_{\mathbf{0}}\right.$, where $\gamma$ is the gyromagnetic ratio, a constant specific to the atomic nucleus), (Figure 1(d)).

This can be explained by a vector diagram of the precessing hydrogen nucleus where at any given instant the net magnetic moment of the nucleus is along the direction of the external magnetic field $\mathrm{B}_{0}$ (Figure 2).

Figure 2
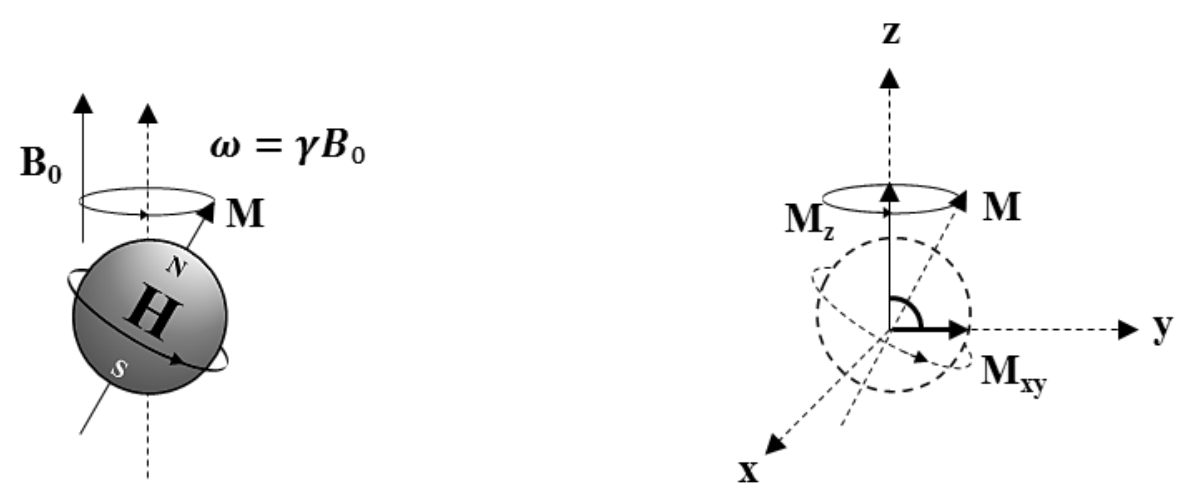

\subsubsection{Basic Components of an MRI Machine}


The basic components of an MRI machine are shown in Figure 3 and include the primary magnet, secondary gradient coil magnets, radiofrequency induction coils, and radiofrequency detectors connected to a computer system (Yan et al. 2010).

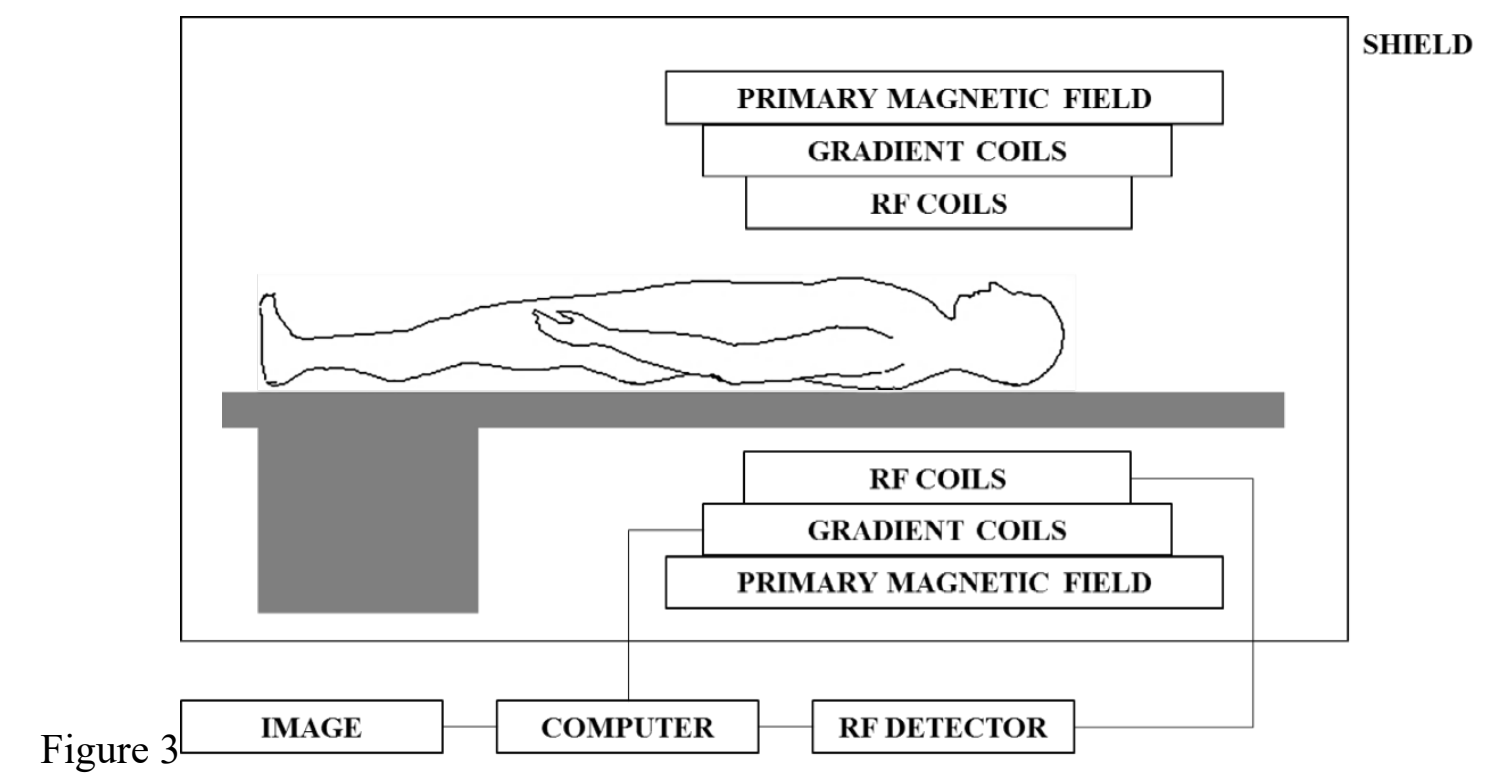

The first component is the superconducting primary magnet that creates a strong, static, permanent magnetic field. The primary field strength of a typical clinical MRI machine is from 0.5-3 Tesla. The second component is a set of three secondary gradient magnets that are capable of producing gradient magnetic fields in the $\mathrm{x}, \mathrm{y}$, and $\mathrm{z}$ axes that allow selection of sections or 'slices' chosen along these planes. The images obtained from specific slices along different axes are therefore termed as axial (z-axis), coronal (y-axis), and sagittal (x-axis) images. The secondary coils can also be programmed to incite signals or responses from cubic volumes or voxels that can be processed to create $3 \mathrm{D}$ images. The third component is a set of radiofrequency (RF) induction coils and detectors that bombard the chosen slice with short-lived electromagnetic radiation or RF pulses. The RF pulses incite a response or signal from the sample, which is also in the radiofrequency range and is detected by $\mathrm{RF}$ detectors placed close to the sample.

To obtain an MR image in a chosen area of interest in the sample, the RF coils produce a radiofrequency pulse at 900 to $\mathrm{B}_{0}$. The magnitude of this RF pulse equals the Larmor precession or resonance frequency of hydrogen atoms and only lasts a few moments. Because of the RF pulse, energy is added to the system which temporarily changes the direction of nuclear spins in one of the following ways (See Figure 4):

(i) the spins of some hydrogen nuclei flip to a high energy state and align antiparallel to $\mathrm{B}_{0}$. This results in a decrease in the longitudinal magnetization. 
(ii) when an RF pulse is applied, the nuclei start to precess in phase with each other, which results in resonance causing a net magnetization, $\mathrm{B} 1$, orientated at $90^{\circ}$ to $\mathrm{B}_{0}$, and rotating about the $\mathrm{B}_{0}$ field. This is termed as transverse magnetization (Mastrogiacomo et al. 2019).
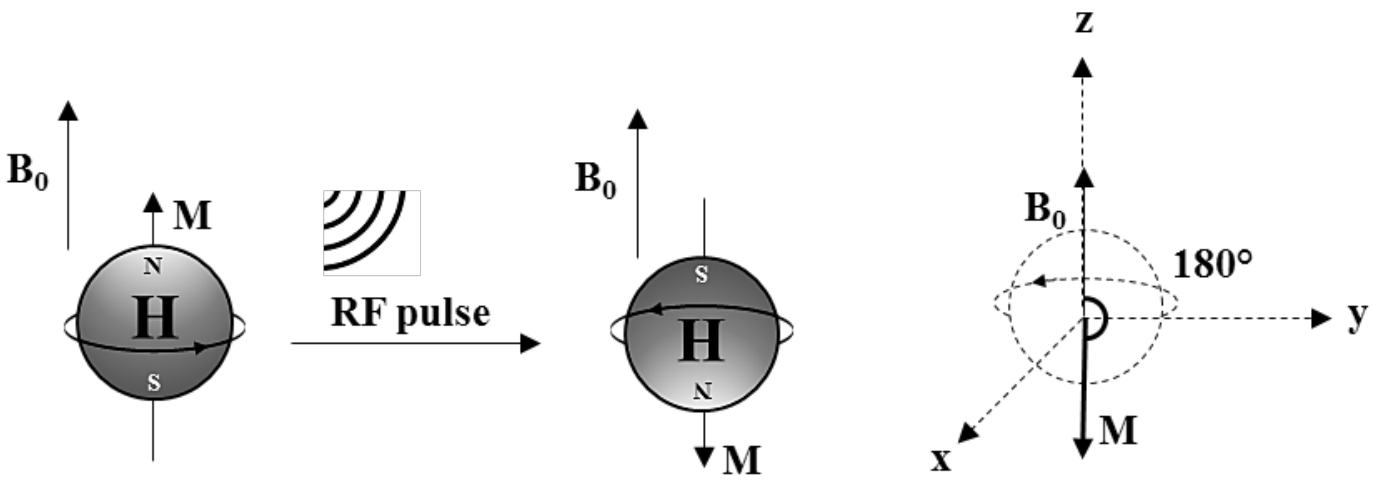

Figure 4
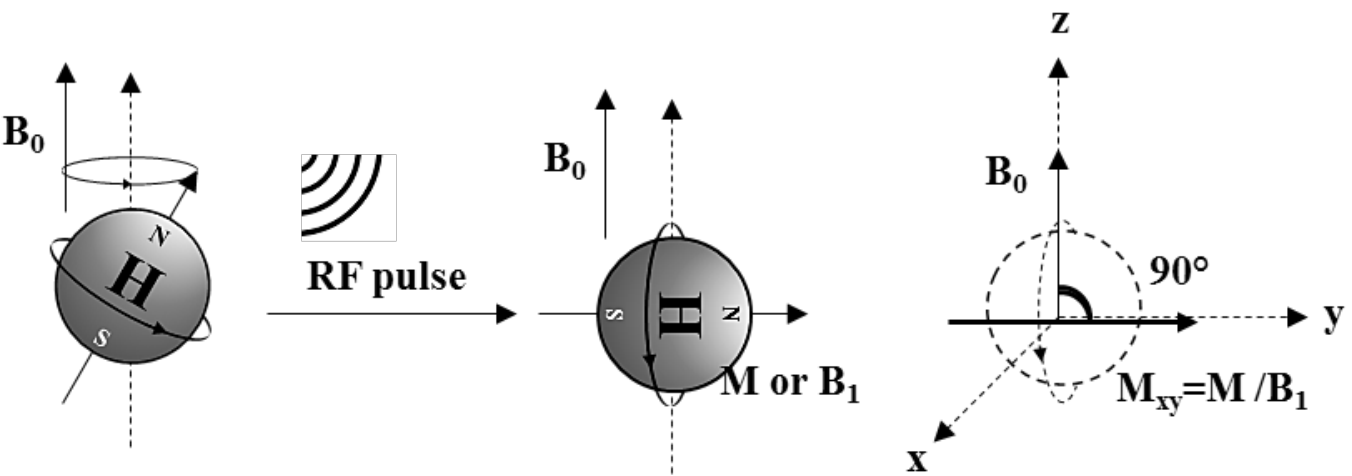

As soon as the RF pulse stops, the nuclei tend to return to their lower energy states by re-adjusting their spins and releasing the absorbed energy. This is termed as relaxation (see Figure 5) and the released energy is detected by the RF detector coils in the machine. Two main types of signals can be observed.

(i) the nuclear spins revert to their original low energy positions relative to $\mathrm{B}_{0}$ and release the absorbed RF energy into the surrounding lattice. This is termed as the spin-lattice relaxation (or longitudinal relaxation) and results in the recovery of the longitudinal magnetization (Mastrogiacomo et al. 2019). This signal is measured along the z-axis or $\mathrm{B}_{0}$ and is termed as a $\mathrm{T} 1$ signal. The T1 signal increases with time and the time taken by $63 \%$ hydrogen nuclei to re-align with the field is termed as T1 relaxation (Mastrogiacomo et al. 2019). The image acquired by the processing of the $\mathrm{T} 1$ signal is termed as a T1-weighted image.

(ii) the precessing nuclei also tend to re-align with $\mathrm{B}_{0}$ and the interaction of spins in an out-of-phase manner results in loss of transverse magnetization and release of absorbed RF energy. This is termed as transverse relaxation or spin-spin relaxation and the signal is termed T2 signal. The T2 signal is detected at $90^{\circ}$ to $\mathrm{B}_{0}$ and is indicative of the reversal of transverse magnetization. The intensity of the T2 signal decreases with time and the time taken by a T2 signal to completely 
decay is termed as $\mathrm{T} 2$ relaxation. The image acquired by the processing of the $\mathrm{T} 2$ signal is termed as a T2-weighted image (Mastrogiacomo et al. 2019).

In order to generate tissue contrast, the duration of RF pulse along with the repetition times (or TR, i.e. the time between two RF pulses) and echo times (or TE, i.e. the time between RF pulse and detection of peak signal) controls image quality.

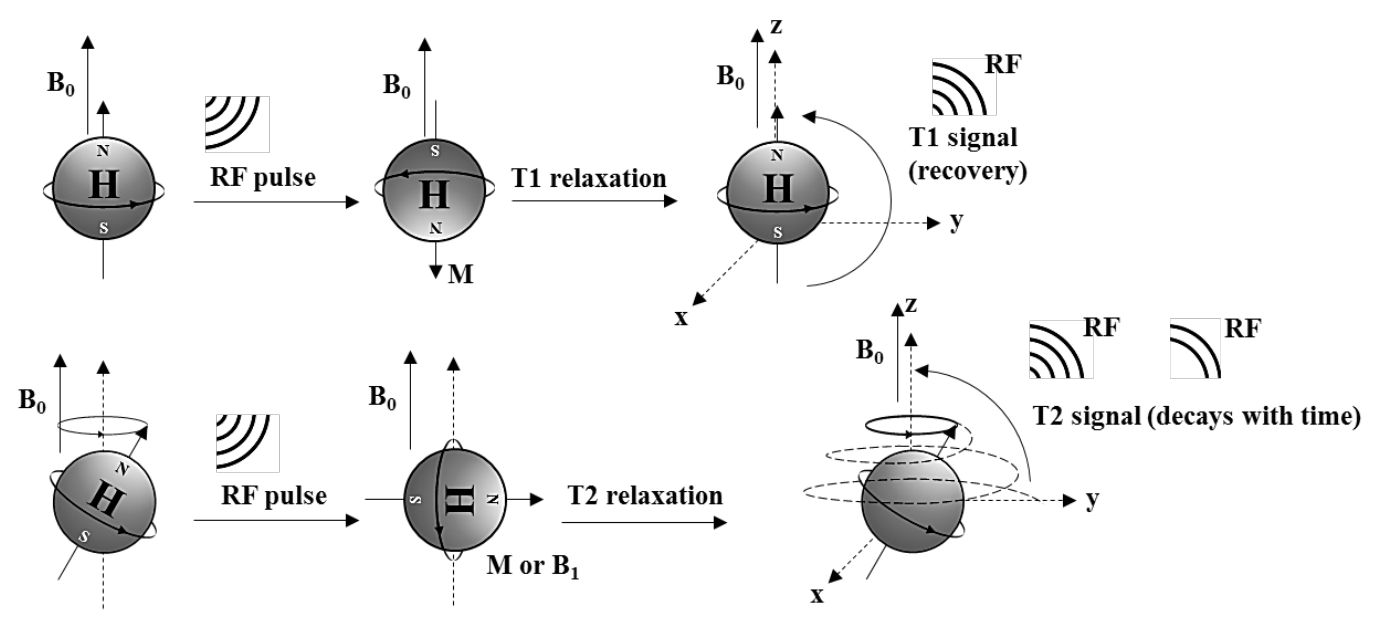

Figure 5:

For T1-weighted images, short TR and TE are required. A short TR value means that the RF excitation pulse is repeated rapidly. As a result, rapid changes in longitudinal magnetization are observed which leads to a greater intensity of the T1 signal. Moreover, the contrast is due to the difference in $\mathrm{T} 1$ recovery times of hydrogen nuclei associated with different molecules like fats and water. Usually, hydrogen nuclei in water molecules have longer T1 relaxation than fats so they appear dark in T1-weighted images (Yoshikawa et al. 2016).

For T2-weighted images, long TR and TE are required and the observed contrast is due to the difference in T2 decay times of hydrogen nuclei associated with different molecules. The local field inhomogeneity created due to the interaction of spins of neighboring protons also results in $\mathrm{T} 2$ relaxation. Since hydrogen nuclei in water molecules have long T2 relaxation times so water-based fluids appear brighter in T2-weighted images (Chavhan et al. 2009).

The RF detector coils collect the T1 or T2 signals and relay it to a computer system that stores this information and then converts them in a pixel-by-pixel image (or a voxel by voxel image in case of 3D images). Both $\mathrm{T} 1$ and $\mathrm{T} 2$ images are equally useful and provide information of a slightly different nature. T1 images usually highlight the distribution of fat content that appears bright and T2 images highlight the distribution of water molecules and fluids (Yan et al. 2010). 
A standard clinical MRI has a resolution in the $\mathrm{mm}$ to sub $\mathrm{mm}$ range. This resolution provides sufficient detail to observe tissue and organ morphology and point out any anomaly in comparison with normal tissues but small changes in physiology like minor inflammation can be disregarded as an artifact due to a low signal-to-noise ratio. Contrast agents are molecules that enhance the sensitivity of an image and further improve image resolution (Chavhan et al. 2009).

\subsubsection{Development of Contrast Agents for MRI}

As described in the previous section, several approaches have been reported in the literature to significantly enhance the sensitivity of MRI. Of these approaches, the development and use of exogenous contrast agents provide a means to improve resolution without changing the instrumentation in any way. As a general rule of thumb, the sensitivity of a molecular imaging probe depends on the distribution of the probe in the intra and extracellular space, the type and intensity of the probe signal (T1 or T2), and the choice of imaging sequence (TR and TE values). To date, a number of contrast agents have been investigated for their ability to generate contrast in live subjects in a non-toxicological manner. These contrast agents can be categorized by their mechanism of action, by their chemical nature and magnetic properties. For in vivo imaging, factors like the concentration of the contrast agent, its toxicity, and retention time in the target tissue along with the speed and mechanism of clearance also play an important role (Hu 2020).

Paramagnetic materials, by their nature, become magnetized in the presence of an external magnetic field and lose their magnetization when the external field is removed. In case of use of these materials as MRI contrast agents, they provide local sites to the hydrogen nuclei where they increase the rate of their magnetic relaxation (thereby reducing $\mathrm{T} 1$ or $\mathrm{T} 2$ relaxation times) by interfering with their magnetic moments. On the other hand, superparamagnetic materials show a higher magnetic susceptibility as compared to paramagnetic materials, which in turn results in a far greater contrast generation. Superparamagnetism is a consequence of size where materials with dimensions in a nano range (1-100 nm) show enhanced magnetic properties.

Paramagnetic materials were one of the earliest materials to be used as contrast agents for MRI. The use of manganese ion and its complexes, stable nitroxide free radicals, and molecular oxygen for contrast enhancement has also been investigated. They also carried out detailed toxicity studies and clearance patterns of these materials (Pan et al. 2011).

The most common classification of contrast agents is the division into T1 and T2 contrast agents. $\mathrm{T} 1$ contrast agents are usually paramagnetic molecules that increase $\mathrm{T} 1$ recovery of hydrogen nuclei in water. The T1 contrast agents have specific sites that attract and bind water molecules, 
allow their protons to undergo $\mathrm{T} 1$ relaxation, and release them at a very fast rate. $\mathrm{T} 1 \mathrm{contrast}$ agents appear bright in MR images.

T2 contrast agents are usually superparamagnetic particulate aggregates that enhance T2 contrast by decreasing $\mathrm{T} 2$ relaxation time by providing a strong magnetic field to the water protons that increases the spin-spin relaxation rates. T2 contrast agents appear dark in MR images (Chavhan et al. 2009). Current trends in research on contrast agents are based on three major groups of contrast agents. The first group includes chelates of paramagnetic gadolinium, termed as the gadoliniumbased contrast agents (GBCA) which are usually T1 contrast agents. The GBCA increases both the T1 recovery and T2 decay by influencing the magnetic moments of water hydrogens through the seven unpaired electrons in the $\mathrm{Gd}^{+3}$ ion. The two signals may interfere with each other so a strong T1 signal is achieved through small TR and TE values (Splendiani et al. 2019).

The second group of contrast agents is based on superparamagnetic iron oxide nanoparticles $\left(\mathrm{Fe}_{3} \mathrm{O}_{4} / \gamma-\mathrm{Fe}_{2} \mathrm{O}_{3}\right)$. The superparamagnetic behavior results in a greater sensitivity at low concentrations. As with most nanoscale structures, surface modification becomes a necessity in designing iron oxide-based contrast agents to prevent agglomeration. This is usually achieved by the introduction of a polymeric coating. The polymeric coatings also allow for the addition of novel functionalities to the contrast agents (Yin et al. 2019).

The third group of contrast agents is based on the hydrogen nuclei associated with water molecules that are chemically exchangeable with the hydrogen atoms in the contrast agents using saturation transfer mechanisms. These agents are termed as CEST (chemical-exchange-dependent saturation transfer) agents and usually cause a decrease in the local water-based signal which becomes visible in an MRI (Zhou et al. 2017).

\subsection{Development of MRI as a Tool for Ultrasensitive Molecular Imaging}

MRI is successfully being used for in vivo imaging of specific cells and molecular targets, however, live imaging can result in artifacts due to interference with the respiratory and cardiac rhythms of the live subject during imaging. This can limit the use of the technique for imaging of molecular targets present in small areas and low concentrations despite the ability of the technique to produce high-resolution images( $\mathrm{Hu} 2020)$. A logical alternative to overcome this limitation is in vitro imaging of excised tissue biopsies or cell suspensions using specific ultrasensitive molecular probes that can lead to the development of MRI as a tool for in vitro diagnostics.

The use of MRI as a molecular imaging modality with superparamagnetic iron oxide-based contrast agents for point-of-care diagnostic applications in an in vitro setting is investigated hereafter. 
The conventional techniques previously demonstrated for their molecular imaging capabilities involve PET, optical imaging, ultrasound, and MRI. Each technique has its advantages and disadvantages in terms of resolution, depth, cost, and sensitivity. MRI can be used as a relatively low-cost molecular imaging technique because it offers a high spatial resolution of target molecules present in deep tissues. The only limiting factors are the sensitivity of the technique and a rather long image acquisition time, which can be overcome through the use of ultrasensitive molecular probes and a dynamic sensing platform that allow for quick surface-based imaging (Brodoehl et al. 2020).

\subsubsection{Development of Iron Oxide-Based Contrast Agents for}

\section{Ultrasensitive Imaging}

In comparison with the GBCA, iron oxide-based contrast agents produce images with significantly high signal-to-noise ratio and higher sensitivity (Splendiani et al. 2019).

Magnetically engineered iron oxide nanoparticles can be synthesized using a number of high temperature, organic synthesis routes, and low temperature, water-based synthesis methods. Advantages such as tunable magnetism with added features such as biocompatibility and specificity allow the use of these materials as contrast agents for ultrasensitive molecular imaging in in vivo systems as systems (Wu et al. 2019). Iron oxide nanoparticles are usually designed with a coreshell structure where the size of the magnetic core has a direct influence on imaging capability and magnetic behavior of these particles and also influences the ability of the particles to be taken up by different types of tissues and cells and their retention time in the blood (Fernández et al. 2020).

In terms of size, the iron oxide-based contrast agents can be divided into three main groups; micrometer-sized paramagnetic iron oxide (MPIO) particles (with a diameter of up to several micrometers), superparamagnetic iron oxide (SPIO) particles (with a diameter of $50 \mathrm{~nm}$ to $500 \mathrm{~nm}$ ) and ultra-small superparamagnetic iron oxide (USPIO) particles (with a diameter less than $50 \mathrm{~nm}$ ) (Song et al. 2019; Yin et al. 2019). To use one of these particles for in vitro cellular visualization applications, the particles should have the ability to be readily taken up by the cells and insufficient concentrations to generate adequate contrast. All three types of iron oxide-based contrast agents have been tested for cellular imaging and it has been demonstrated that while USPIO shows a greater signal enhancement, SPIO and MPIO are better suited for in vitro imaging. As compared to USPIO, lesser concentrations of SPIO and MPIO are readily taken up by the cells to generate sufficient contrast whereas USPIO are required in a greater concentration and also show a limited uptake. Ultrasmall iron oxide nanoparticles (USIONPs) have been recently developed as labeling 
probes for T2 magnetic resonance imaging contrast agents with enhanced cell proliferation and cellular uptake (Park et al. 2020).

Many different core-shell iron oxide nanoparticles have been synthesized where polymeric and non-polymeric, organic and inorganic molecules have been used for surface modification both during and after synthesis for the introduction of desirable properties like biocompatibility, the introduction of targeting ligands and prevention of agglomeration (Natarajan et al. 2019).

Several studies have been carried out to evaluate contrast enhancement of core-shell iron oxide nanoparticles and the effect of different types of polymer coatings on contrast generation has been studied (Wu et al. 2020). Theranostic nanoplatforms with iron oxide NPs are normally utilized as T2 (negative) MRI contrast agents (Gauger et al.2020). In all cases, the magnetic behavior and T2 relaxation rates are governed by the size and composition of magnetic core whereas different types of polymeric shells impart specific surface characteristics to the polymer magnetic particles, like hydrophilic or hydrophobic behavior, tissue specificity, and colloidal stability. In addition to the use of MR imaging for detection of morphological abnormalities in cells, diagnostic magnetic resonance (or DMR) is an up and coming field where highly sensitive detection assays are being developed using SPIO contrast agents that allow detection of specific target molecules through the concept of magnetic resonance switching. These assays are based on the pretext that the presence of certain molecules in an analyte can result in a change in the T2 signal intensity by influencing the aggregation and subsequent T2 relaxation times of SPIO contrast agents that can be correlated to the quantities of these molecules. A number of different detection assays have been developed that allow nanoscale materials combined with various engineered biological molecules (e.g., proteins, enzymes, oligonucleotides, polysaccharides, lipids, biological cofactors and ligands to detect biomolecules like oligonucleotides, proteins, enzymes, carbohydrates, tumor cells, and even viruses and bacteria (Nagamune 2017).

\subsubsection{Development of an Imaging Platform for MRI}

General protocols for MR imaging of cells are designed after the concept of MRI phantoms. These methods are easy to perform in a lab but the sample preparation steps may become cumbersome when MRI is being used for point-of-care DMR applications. Most in vitro DMR assays require free interaction of contrast agents with a target molecule and the difference in signal intensity is based on a change in dispersity or agglomeration of the contrast agent. DMR technology encompasses numerous assay configurations and sensing principles, and to date, magnetic nanoparticle biosensors have been designed to detect a wide range of targets including DNA/mRNA, proteins, enzymes, drugs, pathogens, and tumor cells with exquisite sensitivity 
(Haun et al. 2011). The development of disposable, cheap, and dynamic platforms for MRI that actively generates contrast for fast ultrasensitive molecular imaging is relatively a novel area of research. Immobilization of specific quantities of contrast agents tailored for a specific target molecule onto a substrate can allow for a quick surface-based imaging method where the change in signal intensity is due to adherence of the immobilized contrast agent with the target that limits its interaction with water molecules in solution.

\subsubsection{Electrostatic Layer-by-Layer Self Assembly for Magnetic Thin}

\section{Films}

Electrostatic layer-by-layer (LbL) self-assembly is a simple, economic method to create thin films of desired components based on electrostatic attraction between two or more oppositely charged components. For thin film fabrication, the LbL self-assembly technique has certain advantages in comparison with other contemporary deposition techniques such as vacuum deposition, solvent casting, spin coating, and Langmuir-Blodgett (LB) deposition. The requirement of expensive instrumentation, lengthy time-consuming processes along with requirements of specific substrates, and the formation of thick non-uniform films are some of the disadvantages that hinder these techniques. Layer-by-layer self-assembly on the other hand provides a robust, environmentally friendly alternative that requires no expensive instrumentation and can be used for the deposition of uniform multilayers on a diverse range of substrates (Keeney et al. 2015). Since the starting material in LbL self-assembly is a charged substrate, the choice of substrate and surface charge of the substrate also considerably affects the growth of thin films. A diverse range of substrates can be used for LbL self-assembly including silicon wafers, mica, and glass. Moreover, thin films deposited on such substrates via other techniques can also be used as substrates for LbL selfassembly. It is based on the concept, where two oppositely charged species can be sequentially adsorbed onto a charged substrate leading to the formation of a bi-layer composite. The process can be repeated to obtain multiple bilayer composites of up to hundreds of bilayers (Keeney et al. 2015). Moreover, more charged components could be added to create films of complex multilayered architectures with compound properties. Furthermore, a wide variety of selfassembled thin films can be fabricated ranging from polyelectrolytes and colloids to proteins and DNA, solely based on surface charge (Keeney et al. 2015). 


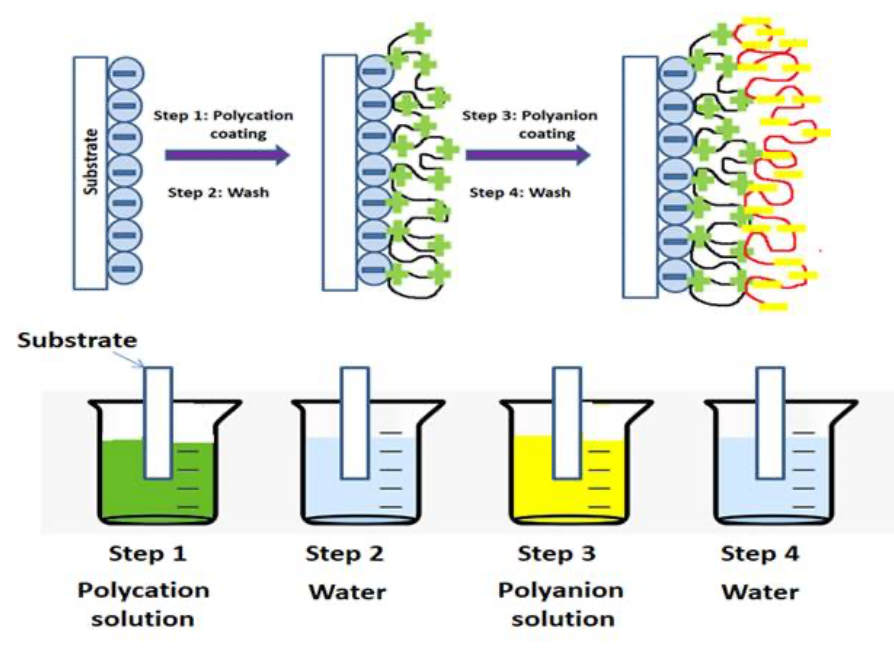

Figure 6

Besides the predominant electrostatic attraction between oppositely charged species, many other molecular interactions at the bilayer interface also influence the growth of layer-by-layer selfassembled multilayers (LbL-SAMu) thin films such as hydrogen bonding, hydrophobic interactions, and van der Waals interactions (Rawtani and Agrawal 2014). Moreover, the growth of multilayer thin films can also be explained based on the charge overcompensation phenomenon. Charge overcompensation refers to adsorption of a greater amount of an adsorbing electrolyte onto a previously adsorbed layer of the opposite charge leading to the reversal of surface charge rather than resulting in the neutralization of the previous charge. This charge inversion effect increases the amount of net surface charge on the top layer during the formation of subsequent bilayers and as a result the quantity of adsorbing electrolytes also increases considerably with an increase in the number of bilayers (Keeney et al. 2015).

The fabrication conditions like salt, $\mathrm{pH}$, and temperature also influence thin film growth if the components involved are susceptible to particular conditions. Varying fabrication conditions like salt concentration, $\mathrm{pH}$, and temperature can lead to the formation of stimuli-responsive thin films that change their features in response to a change in these conditions. Wide arrays of stimuliresponsive thin films have been fabricated in which salt, $\mathrm{pH}$, or temperature-responsive polyelectrolytes are used in combination with dyes, enzymes, and drugs. Such films find applications in areas like sensing, diagnostics, and drug delivery (Park et al. 2018; Rawtani and Agrawal 2014)

In addition to changing fabrication conditions, the stimuli-responsive films can also be fabricated by the specific properties of the components involved in the film, for example, thin films capable of responding to environmental stimuli such as light (Shaikh et al. 2014) and magnetic field (Paterno et al. 2009) can also be fabricated. The ability to add structurally diverse components with specific properties alongside stimuli-responsive behavior can lead to the utilization of LbL-SAMu thin 
films for a wide range of applications, such as the introduction of magnetically active components for fabrication of magnetic thin films.

Gorin et al., developed LbL-SAMu thin films of polyethyleneimine and iron oxide nanoparticles onto silicon wafer substrates and elucidated their optical and magnetic properties. They observed that properties like refractive index electron magnetic resonance vary with the adsorbed volume fraction of iron oxide nanoparticles, which in turn relates directly with the number of bilayers. The results indicate the controllability of optical and magnetic properties by varying the number of bilayers in the films (Gorin et al. 2009).

Paterno et al., developed electrically and magnetically active LbL-SAMu thin films using poly(oethoxyaniline) (POEA), sulfonated polystyrene (PSS) and positively-charged maghemite nanoparticles onto glass substrates. The electrical and the magnetic measurements indicated successful incorporation of maghemite nanoparticles into the films which exhibited a dual functionality i.e. electrical conductivity and magnetic properties and prospective uses of such films in electromagnetic interference shielding and chemical sensing were suggested (Paterno et al. 2009).

Among other uses, the magnetic properties of magnetically engineered LbL-SAMu films were specifically utilized in surface-based MR imaging. The utilization of layer-by-layer self-assembly technique for the synthesis of magnetically engineered thin films of contrast agents for surfacebased MR imaging was described by Hassan et al. where thin films of nickel ferrite nanoparticles and chitosan were fabricated onto microscopic glass slides using a manual fabrication protocol. The thin films were fabricated with a different number of bilayers per sample, which resulted in the different number of particles adsorbed on the top layer. These samples were tested for their ability to generate a difference in intensity using a thin film of blood as a test liquid, but the T1 and T2weighted images were not described in detail (Hassan et al. 2013).

Similarly, thin films of spherical negatively charged core-shell magnetic latex particles consisting of PDAC were fabricated using the layer-by-layer self-assembly approach by Ahsan et al. (Ahsan et al. 2013) and characterized through SEM and AFM. The films showed adsorption of spherical particles as is without any change in particle morphology. The aqueous solutions of colloids were tested for their contrast-enhancing behavior, which revealed them, T2 contrast agents. The magnetic behavior of the films was, however, not tested (Ahsan et al. 2013). Thin film gradients of negatively charged magnetic colloidal particles and PDAC were fabricated using the layer-by-layer self-assembly techniques and their diagnostic capability was evaluated by obtaining T1- and T2weighted images using water as the test liquid. Film gradients showed a decreasing trend in signal intensity of T2 weighted images with an increase in the number of bilayers. These gradient films 
can be used as a dipstick approach in routine clinical diagnosis (Khizar et al. 2020).

\subsection{Conclusions and Final Remarks}

This chapter reports on magnetic resonance imaging (MRI) with a special focus on its development as a technique for ultrasensitive molecular imaging. In the first part, the fundamentals of MRI are introduced and its basic components are presented succinctly. Throughout this part, the general advantages and drawbacks of this technique are discussed compared to that of other imaging techniques. Recent scientific progress in the field of MRI is targeted at improving significantly its sensitivity. Hence, the development of relevant contrast agents may overcome sensitivity issues without changing the instrumentation. One of the promising candidates is iron oxide and its derivatives.

The second part is dedicated to the utilization of MRI as a technique for ultrasensitive molecular imaging through the development of combinatorial thin film gradients that can be used as a substrate for in vitro imaging of fluidic biological samples like blood, or suspensions of cells and cell lysates. Their fabrication is investigated through an electrostatic layer-by-layer self-assembly technique. The proposed materials chip is composed of a gradient thin film of colloidal core-shell magnetic nanoparticles that serve as exogenous contrast agents to generate a negative contrast in aqueous environments in an MRI. The unique core-shell morphology of these colloids coupled with adequately tailored magnetic properties can significantly increase the signal-to-noise ratio and consequently, the sensitivity of the MR images. The development of thin film gradients will eliminate sample preparation steps and allow the development of a disposable chip for dipstick like approach toward molecular diagnostics.

\section{Acknowledgments}

MS Thesis Chapter 1 and 2: Title of Thesis: Combinatorial Lab-on-chip Self-assembled Thin Films of Magnetic Polymer Colloids for Ultrasensitive Molecular Imaging, MS student name: Sundas Khalid, Supervisor Dr. Nasir M. Ahmad; MS Thesis April 2016, School of Chemical and Materials Engineering (SCME), National University of Sciences and Technology (NUST), Islamabad, Pakistan.

\section{References}

Ahsan, A., Aziz, A., Arshad, M. A., Ali, O., Nauman, M., Ahmad, N. M., \& Elaissari, A. (2013). Smart Magnetically Engineering Colloids and Biothin Films for Diagnostics Applications. Journal 
of Colloid Science and Biotechnology, vol. 2(1), pp.19-26. doi: 10.1166/jcsb.2013.1031

Atabo, S. M., \& Umar, A. A. (2019). A review of imaging techniques in scientific research/clinical diagnosis. MOJ Anatomy \& Physiology, vol.6(5), pp.175-183. doi: 10.15406/mojap.2019.06.00269

Brodoehl, S., Gaser, C., Dahnke, R., Witte, O. W., \& Klingner, C. M. (2020). Surface-based analysis increases the specificity of cortical activation patterns and connectivity results. Scientific reports, vol.10(1), pp.5737. doi: 10.1038/s41598-020-62832-z

Chavhan, G. B., Babyn, P. S., Thomas, B., Shroff, M. M., \& Haacke, E. M. (2009). Principles, techniques, and applications of $\mathrm{T} 2 *$-based $\mathrm{MR}$ imaging and its special applications. Radiographics : a review publication of the Radiological Society of North America, vol.29(5), pp.1433-1449. doi: 10.1148/rg.295095034

Du, S., Wu, J., AlShareedah, O., \& Shi, X. (2019). Nanotechnology in Cement-Based Materials: A Review of Durability, Modeling, and Advanced Characterization. Nanomaterials (Basel), vol. 9(9), pp.1213. doi: 10.3390/nano9091213

Fernández-Barahona, I., Muñoz-Hernando, M., Ruiz-Cabello, J., Herranz, F., \& Pellico, J. (2020). Iron Oxide Nanoparticles: An Alternative for Positive Contrast in Magnetic Resonance Imaging. Inorganics, vol.8(4), pp.28. doi: 10.3390/inorganics8040028

Fiordelisi, M. F., Cavaliere, C., Auletta, L., Basso, L., \& Salvatore, M. (2019). Magnetic Resonance Imaging for Translational Research in Oncology. Journal of clinical medicine, vol.8(11),pp.1883. doi: 10.3390/jcm8111883

Gauger, A. J., Hershberger, K. K., \& Bronstein, L. M. (2020). Theranostics Based on Magnetic Nanoparticles and Polymers: Intelligent Design for Efficient Diagnostics and Therapy. Frontiers in chemistry, vol.8, pp.1-7. doi: 10.3389/fchem.2020.00561

Geraldi, A., \& Giri-Rachman, E. A. (2019). Synthetic biology-based portable in vitro diagnostic platforms. Alexandria Journal of Medicine, vol.54(4), pp.423-428. doi: 10.1016/j.ajme.2018.11.003

Gordon, O., Ruiz-Bedoya, C. A., Ordonez, A. A., Tucker, E. W., \& Jain, S. K. (2019). Molecular Imaging: a Novel Tool To Visualize Pathogenesis of Infections In Situ. mBio, vol.10(5),pp. e00317-19.doi: 10.1128/mBio.00317-19

Gorin, D. A., Yashchenok, A. M., Koksharov, Y. A., Neveshkin, A. A., Serdobintsev, A. A., Grigoriev, D. O., \& Khomutov, G. B. (2009). Surface morphology and optical and magnetic properties of polyelectrolyte/magnetite nanoparticles nanofilms. Technical Physics, vol.54(11), pp.1675-1680. doi: 10.1134/s1063784209110206

Grob, U., Krass, M. D., Heritier, M., Pachlatko, R., Rhensius, J., Kosata, J., . . . Degen, C. L. (2019). Magnetic Resonance Force Microscopy with a One-Dimensional Resolution of 0.9 Nanometers. Nano letters, vol.19(11), pp.7935-7940. doi: 10.1021/acs.nanolett.9b03048

Hassan, M. A., Saqib, M., Shaikh, H., Ahmad, N. M., \& Elaissari, A. (2013). Magnetically Engineered Smart Thin Films: Toward Lab-on-Chip Ultra-Sensitive Molecular Imaging. Journal 
of biomedical nanotechnology, vol.9(3), pp.467-474. doi: 10.1166/jbn.2013.1562

Haun, J. B., Yoon, T. J., Lee, H., \& Weissleder, R. (2011). Molecular detection of biomarkers and cells using magnetic nanoparticles and diagnostic magnetic resonance. Methods in molecular biology,vol. 726, pp.33-49. doi: 10.1007/978-1-61779-052-2_3

Hu, H. (2020). Recent Advances of Bioresponsive Nano-Sized Contrast Agents for Ultra-HighField Magnetic Resonance Imaging. Frontiers in chemistry, vol.8, pp.203. doi: 10.3389/fchem.2020.00203

Keeney, M., Jiang, X. Y., Yamane, M., Lee, M., Goodman, S., \& Yang, F. (2015). Nanocoating for biomolecule delivery using layer-by-layer self-assembly. Journal of materials chemistry. B, vol.3(45), pp.8757-8770. doi: 10.1039/c5tb00450k

Khalid, K., Tan, X., Mohd Zaid, H. F., Tao, Y., Lye Chew, C., Chu, D. T., . . . Chin Wei, L. (2020). Advanced in developmental organic and inorganic nanomaterial: a review. Bioengineered, vol.11(1),pp. 328-355. doi: 10.1080/21655979.2020.1736240

Khizar, S., Ahmad, N. M., Saleem, H., Hamayun, M. A., Manzoor, S., Lebaz, N., \& Elaissari, A. (2020). Magnetic Colloidal Particles in Combinatorial Thin-Film Gradients for Magnetic Resonance Imaging and Hyperthermia. Advances in Polymer Technology, vol.2020, pp.1-18. doi: $10.1155 / 2020 / 7163985$

Mastrogiacomo, S., Dou, W., Jansen, J. A., \& Walboomers, X. F. (2019). Magnetic Resonance Imaging of Hard Tissues and Hard Tissue Engineered Bio-substitutes. Molecular imaging and biology, vol.21(6), pp.1003-1019. doi: 10.1007/s11307-019-01345-2

Nagamune, T. (2017). Biomolecular engineering for nanobio/bionanotechnology. Nano convergence, vol.4(1),pp. 9. doi: 10.1186/s40580-017-0103-4

Natarajan, S., Harini, K., Gajula, G. P., Sarmento, B., Neves-Petersen, M. T., \& Thiagarajan, V. (2019). Multifunctional magnetic iron oxide nanoparticles: diverse synthetic approaches, surface modifications, cytotoxicity towards biomedical and industrial applications. BMC Materials, vol.1(1), pp.2. doi: 10.1186/s42833-019-0002-6

Pan, D., Schmieder, A. H., Wickline, S. A., \& Lanza, G. M. (2011). Manganese-based MRI contrast agents: past, present and future. Tetrahedron, vol.67(44), pp.8431-8444. doi: 10.1016/j.tet.2011.07.076

Park, K., Choi, D., \& Hong, J. (2018). Nanostructured Polymer Thin Films Fabricated with Brush-based Layer-by-Layer Self-assembly for Site-selective Construction and Drug release. Scientific reports, pp.8(1), pp.3365. doi: 10.1038/s41598-018-21493-9

Park, S., Sherwood, J. A., Hauser, R. M., Antone, A. J., Beswick, B. T., Lubin, F. D., . . Kim, Y. (2020). Surface Effects of Ultrasmall Iron Oxide Nanoparticles on Cellular Uptake, Proliferation, and Multipotency of Neural Stem Cells. ACS Applied Nano Materials, vol.3(2), pp.1542-1552. doi: $10.1021 /$ acsanm.9b02331

Paterno, L. G., Fonseca, F. J., Alcantara, G. B., Soler, M. A. G., Morais, P. C., Sinnecker, J. P., . . . Mattoso, L. H. C. (2009). Fabrication and characterization of nanostructured conducting polymer films containing magnetic nanoparticles. Thin Solid Films, vol.517(5), pp.1753-1758. doi: 
Rawtani, D., \& Agrawal, Y. K. (2014). Emerging Strategies and Applications of Layer-by-Layer Self-Assembly. Nanobiomedicine, vol.1,pp. 8. doi: 10.5772/60009

Shaikh, H., Hassan, M., \& Ahmad, N. M. (2014). Stimuli-responsive self-assembled multilayer azo thin films: Photo-switchable absorbance and morphological characteristics. Photonics and Nanostructures - Fundamentals and Applications, vol.12(1), pp.34-44. doi: 10.1016/j.photonics.2013.07.010

Simonsen, A., Sanchez-Heredia, J. D., Saarinen, S. A., Ardenkjaer-Larsen, J. H., Schliesser, A., \& Polzik, E. S. (2019). Magnetic resonance imaging with optical preamplification and detection. Scientific reports,vol. 9(1), pp.18173. doi: 10.1038/s41598-019-54200-3

Song, C., Sun, W., Xiao, Y., \& Shi, X. (2019). Ultrasmall iron oxide nanoparticles: synthesis, surface modification, assembly, and biomedical applications. Drug discovery today,vol. 24(3), pp.835-844. doi: 10.1016/j.drudis.2019.01.001

Splendiani, A., Corridore, A., Torlone, S., Martino, M., Barile, A., Di Cesare, E., \& Masciocchi, C. (2019). Visible T1-hyperintensity of the dentate nucleus after multiple administrations of macrocyclic gadolinium-based contrast agents: yes or no? Insights into imaging, vol.10(1), pp.82. doi: $10.1186 / \mathrm{s} 13244-019-0767-\mathrm{x}$

Ward, A., Walton, K., Mawla, N., Kaialy, W., Liu, L., Timmins, P., . . Asare-Addo, K. (2019). Development of a novel method utilising dissolution imaging for the measurement of swelling behaviour in hydrophilic matrices. International journal of pharmaceutics:X, vol.1, pp.100013. doi: 10.1016/j.ijpx.2019.100013

Wong, X. Y., Sena-Torralba, A., Alvarez-Diduk, R., Muthoosamy, K., \& Merkoci, A. (2020). Nanomaterials for Nanotheranostics: Tuning Their Properties According to Disease Needs. ACS Nano, vol.14(3), pp.2585-2627. doi: 10.1021/acsnano.9b08133

Wu, K., Su, D., Liu, J., Saha, R., \& Wang, J. P. (2019). Magnetic nanoparticles in nanomedicine: a review of recent advances. Nanotechnology, vol.30(50), pp.502003. doi: 10.1088/1361$6528 / \mathrm{ab} 4241$

Wu, Y., Lu, Z., Li, Y., Yang, J., \& Zhang, X. (2020). Surface Modification of Iron Oxide-Based Magnetic Nanoparticles for Cerebral Theranostics: Application and Prospection. Nanomaterials (Basel), vol.10(8), pp.1441. doi: 10.3390/nano10081441

Yan, M., Fresnais, J., \& Berret, J. F. (2010). Growth mechanism of nanostructured superparamagnetic rods obtained by electrostatic co-assembly. Soft Matter, vol.6(9), pp.1997. doi: $10.1039 / \mathrm{b} 919992 \mathrm{f}$

Yin, J., Yao, D., Yin, G., Huang, Z., \& Pu, X. (2019). Peptide-Decorated Ultrasmall Superparamagnetic Nanoparticles as Active Targeting MRI Contrast Agents for Ovarian Tumors. ACS applied materials \& interfaces, vol.11(44), pp.41038-41050. doi: 10.1021/acsami.9b14394

Yoshikawa, T., Tanizawa, A., Suzuki, K., Tanaka, N., Hayashi, T., Tsuda, M., . . Ohshima, Y. (2016). The Usefulness of T1-Weighted Magnetic Resonance Images for Diagnosis of Acute Leukemia Manifesting Musculoskeletal Symptoms prior to Appearance of Peripheral Blood 
Abnormalities. Case reports in pediatrics, vol.2016, pp.2802596. doi: 10.1155/2016/2802596

Zhou, I. Y., Wang, E., Cheung, J. S., Zhang, X., Fulci, G., \& Sun, P. Z. (2017). Quantitative chemical exchange saturation transfer (CEST) MRI of glioma using Image Downsampling Expedited Adaptive Least-squares (IDEAL) fitting. Scientific reports, vol.7(1), pp.84. doi: 10.1038/s41598-017-00167-y

Zubkov, M. A., Andreychenko, A. E., Kretov, E. I., Solomakha, G. A., Melchakova, I. V., Fokin, V. A., . . Slobozhanyuk, A. P. (2018). Ultrahigh field magnetic resonance imaging: new frontiers and possibilities in human imaging. Physics-Uspekhi, vol.189(12), pp.1293-1314. doi: 10.3367/UFNr.2018.12.038505

\section{Figure legends}

iigure 1 Effect of external magnetic field on hydrogen nuclei associated with water molecules. In the figure, (a) Hydrogen nuclei are randomly aligned in the absence of a field. (b) In the presence of an external field $\mathrm{B}_{0}$, the moments of the nuclei align parallel or anti-parallel to the external field. (c) The hydrogen nuclei develop a net magnetic moment (M) parallel to the field (d) Precessional motion of the aligned nuclei.

Figure 2 Vector diagram of the precessing hydrogen nucleus. At any given instant the $\mathrm{Mz}$ component of the magnetic moment $\mathrm{M}$ is with along $\mathrm{B}_{0}$ so the net magnetization of the nucleus is along $\mathrm{B}_{0}$. $\mathrm{Mxy}$ is the component of $\mathrm{M}$ rotating in the xy plane.

Figure 3 Components of a typical clinical MRI machine. The MRI machine consists of a system of primary and gradient magnets, RF coils, and detectors that translate the phenomenon of nuclear magnetic resonance of hydrogen nuclei in a sample and computer-generated image.

Figure 4 Effect of RF pulse on longitudinal and transverse magnetization. An incident RF pulse decreases longitudinal magnetization as (a) it causes the nuclei to align in the antiparallel direction, or (b) causes the magnetic moment of the nucleus to rotate about $\mathrm{B}_{0}$ in the xy-plane. This creates a magnetic moment at $90^{\circ}$ to $\mathrm{B}_{0}$, termed $\mathrm{B} 1$. This is called transverse magnetization.

iigure 5 Longitudinal and transverse relaxation.

Figure 6 Basic concepts of electrostatic layer-by-layer self-assembly. In the figure, the molecular depiction of electrostatic layer-by-layer self-assembly of two oppositely charged polyelectrolytes onto a positively charged substrate. 